\title{
Consistency Analysis of the Simplified Refined Instrumental Variable Method for Continuous-time Systems *
}

\author{
Siqi Pan, Rodrigo A. González, James S. Welsh and Cristian R. Rojas
}

\begin{abstract}
In this paper, we analyse the consistency of the Simplified Refined Instrumental Variable method for Continuous-time systems (SRIVC). It is well known that the intersample behaviour of the input signal influences the quality and accuracy of the results when estimating and simulating continuous-time models. Here, we present a comprehensive analysis on the consistency of the SRIVC estimator while taking into account the intersample behaviour of the input signal. The main result of the paper shows that, under some mild conditions, the SRIVC estimator is generically consistent. We also describe some conditions when consistency is not achieved, which is important from a practical standpoint. The theoretical results are supported by simulation examples.
\end{abstract}

Key words: Consistency, Continuous-time systems, Identification, Instrumental variable method

\section{Introduction}

Direct continuous-time (CT) identification algorithms based on sampled data have achieved remarkable success in many practical applications [25,27,8,9]. In particular, the Refined Instrumental Variable method for Continuous-time systems (RIVC) and its simplest embodiment, the Simplified RIVC (SRIVC) [29] are considered to be the most reliable algorithms in CT system identification $[8,9]$ and have been used in practice for almost 40 years. However, there has been limited theoretical support for these algorithms. Most of the discussions with respect to the properties of these estimators, such as consistency and statistical efficiency, are based on empirical observations [29,25,8,24,23,28]. By providing the user with the theoretical properties of the estimator, they will be better informed as to the conditions necessary to achieve accurate estimates. It is therefore important for any estimator used in practice to have solid theoretical support.

The objective of this paper is to provide the theoretical support in terms of consistency for the SRIVC estimator. Based on the theoretical results, the user will then be able to make conscious decisions when applying this algorithm in practice with respect to obtaining

\footnotetext{
* This paper was not presented at any IFAC meeting.

Email addresses: siqi.pan@uon.edu.au (Siqi Pan), grodrigo@kth.se (Rodrigo A. González), james.welsh@newcastle.edu.au (James S. Welsh), crro@kth.se (Cristian R. Rojas).
}

consistent estimates. There have been some attempts in the existing literature $[11,5,26]$ to examine the consistency property of the SRIVC estimator. However, they are all based on the well-developed theoretical results in discrete-time (DT) system identification [18,17,21,20], which do not provide a mechanism to include the intersample behaviour of the underlying CT system. The current paper analyses the consistency property of the SRIVC estimator while taking into account the intersample behaviour of the signals. The theoretical results we obtain here explicitly inform the user when consistency can be achieved by the SRIVC estimator. The conditions under which the estimator is not consistent are clearly stated as well, and suggestions are also given to alleviate the bias on the estimates in practical situations. We would like to reiterate that if it is important, in practice, to have a consistent estimator, then the theoretical results presented in this paper clearly describe how this can be achieved.

As mentioned above, a significant challenge presented when estimating a CT system is that only sampled inputoutput data are available as measurements. Therefore, the measured input needs to be interpolated in some manner in order to simulate a CT model output. It has been discussed in $[14,1]$ that violating the input intersample behaviour assumption of the underlying data generating process may lead to severe estimation errors. A simple motivation example has been provided in [14] to illustrate the modelling error induced in the estimation process when a band-limited input is assumed while the true system input is a zero-order hold. In some prac- 
tical situations, the input intersample behaviour will be unknown, e.g. environmental modelling, and some interpolation methods have been discussed in [16] to better approximate the input in these cases. We note that if the input is applied and controlled by the user, then it generally can be interpolated exactly. There are, however, some exceptions, e.g. identification of cascaded systems. The input signal to the second system in a cascaded system will be a continuous function of time that cannot be interpolated exactly between samples. Hence, an error will be induced on the modelled output, and this in turn will affect the estimated parameters. Thus, it is important to take into account the intersample behaviour of the signals when dealing with CT estimators, which has been somewhat overlooked in the existing literature. We also note that there are some CT identification methods that do not require the sampled signals to be interpolated. For example, higher order Padé approximation is used in [6] to approximate a DT filter that produces the same output as the sampled CT filter output, which avoids the need to reconstruct the CT input signal, and CT models are identified in [13] based on second-order statistics.

The consistency analysis in the current paper has some similarities to the work in [11] as both analyses are based on the consistency theorem found in [18, Theorem 4.5] developed for the DT bootstrap instrumental variable (IV) method. The work in [11] analyses the convergence of the RIVC estimator with an autoregressive noise model. There are, however, a few shortcomings associated with the analysis in [11]. An extra filter is introduced for the purpose of discretising the derivatives of the input signal, which is not part of the RIVC implementation. We note that this extra filter introduces unnecessary complexity into the analysis, and its role can be replaced by filters that are already part of the algorithm. In addition, due to the formulation of the proof in [11], non-causal filters may arise since the system and model are allowed to be parameterised as biproper transfer functions. Furthermore, the first step to establish the convergence, and therefore the consistency of the RIVC algorithm is to show that a solution of the estimator exists [11]. This relies on 1) the Sylvester matrices constructed from the system and model polynomials being non-singular, and 2) the expectation of the two filtered input vectors, denoted by $\Phi$ in [11], being non-singular. Firstly, in [11], the non-singularity of the Sylvester matrix does not comply with their given assumptions. The analysis assumes that the system and model are parameterised with monic denominator polynomials. It can be shown that this implicit assumption, together with Assumption A5 in [11] on the model order, results in the Sylvester matrix constructed from the system polynomials being singular when the degree of the model denominator is greater than that of the system. The proof of Theorem 1 in [11] with respect to assumption A5 therefore cannot proceed once the Sylvester matrix is singular. Secondly, it is stated in [11] that showing the non-singularity of $\Phi$ relies on the matrix $E\left\{U U^{\top}\right\}$ being non-singular, where $U$ is the vector containing the input samples with sample size $N$ (see (19) in [11]). We note that $E\left\{U U^{\top}\right\}$ has dimension $N \times N$, and is only non-singular up to the order of persistent excitation of the input. Thus, in the asymptotic case, it is not sufficient to conclude that $\Phi$ is always non-singular under the persistent excitation assumption given in [11].

Other work related to consistency such as [5] assumes that the model structure is exactly known and does not take into account the intersample behaviour of the input as part of the analysis. The work in [26] describes a unified Refined Instrumental Variable (RIV) approach for estimating DT or CT transfer functions characterised by a unified operator that can be interpreted in terms of a backward shift, derivative or delta operator. This unified RIV [26] is suggested to be optimal in maximum likelihood, prediction error minimisation and instrumental variable terms under the Box-Jenkins model structure for both discrete and continuous-time. However, only limited theoretical analysis is provided with respect to the consistency of the estimates by using an incremental implementation of the algorithm with no explicit mention of the intersample behaviour of the signal. By neglecting the intersample behaviour as part of the analysis, the results in $[11,26,5]$ have overlooked the possibility that the converging point of the estimator no longer corresponds to the true system parameters when the system input cannot be interpolated exactly.

In this paper, we analyse the consistency property by incorporating the intersample behaviour of the input, the output and the instrument signals. The main result of the paper shows that the SRIVC estimator is generically consistent under some mild conditions in the presence of additive coloured noise on the measured output. In the proof of the consistency theorem, the use of an additional filter, such as in [11], is avoided by discretising the derivatives of the input signal with filters that are already part of the SRIVC implementation. The denominator of the model is parameterised as a non-monic polynomial. This ensures that the Sylvester matrix is always non-singular for model orders satisfying the condition for the existence of a unique solution, i.e. when the model orders are equal to the system orders, or when one of the model polynomial degrees is greater than that of the system. Two common interpolation methods, namely the firstorder hold $(\mathrm{FOH})$ and the zero-order hold $(\mathrm{ZOH})$, are considered for the true system input to conduct the consistency analysis in the main theorem and the corollaries. In addition, in the first part of the consistency theorem where the existence of a solution is shown, we employ the notion of generic consistency [18, Theorem 4.1], i.e. the set of normal matrices of the SRIVC method yield inconsistent estimates has Lebesgue measure zero. This implies that there are rare cases where a certain combination of the input and system parameters can make the normal matrix singular even though all the assump- 
tions are satisfied. We show the generic non-singularity of the normal matrix through the use of analytic functions by following the method presented in [7, Lemma 1]. Furthermore, we have shown that the intersample behaviour of the input in the instrument vector does not influence the consistency of the SRIVC estimator; however, in order for the SRIVC estimator to be generically consistent, the intersample behaviour of the input in the regressor vector must match that of the true system input. We also note that the intersample behaviour of the output in the regressor vector does not impact on the consistency of the SRIVC estimator at the converging point of the iterative algorithm. The practical implications of this paper are to inform the user of the conditions necessary to achieve consistency of the SRIVC estimator and also of the conditions that may lead to an inconsistent estimate.

This paper is organised as follows. Section 2 provides system and model definitions as well as a description of the SRIVC estimator and the definition of generic consistency. This is followed by Section 3, where the theoretical results of the paper, including the consistency theorem and its related corollaries and remarks, are presented. Section 4 provides simulation results that support the theoretical analysis, and the paper is concluded in Section 5.

\section{Preliminaries}

In this section, we define the structure of the continuoustime single-input single-output system and model and provide a brief description of the SRIVC estimator and the definition of generic consistency.

The true system is described as a proper transfer function given by

$$
S:\left\{\begin{array}{l}
\stackrel{\circ}{x}(t)=\frac{B^{*}(p)}{A^{*}(p)} \stackrel{\leftrightarrow}{u}(t) \\
\stackrel{\circ}{y}(t)=\stackrel{x}{x}(t)+v(t),
\end{array}\right.
$$

where the circles $\left({ }^{\circ}\right)$ signify that the signals are associated with the true system. The numerator and denominator polynomials are coprime with degrees given by $m^{*}$ and $n^{*}$ respectively, i.e.

$$
\begin{aligned}
& B^{*}(p)=b_{0}^{*} p^{m^{*}}+b_{1}^{*} p^{m^{*}-1}+\cdots+b_{m^{*}}^{*} \\
& A^{*}(p)=a_{1}^{*} p^{n^{*}}+a_{2}^{*} p^{n^{*}-1}+\cdots+a_{n^{*}}^{*} p+1
\end{aligned}
$$

with $p$ being the differential operator, i.e. $p^{i} x(t)=\frac{d^{i} x(t)}{d t^{i}}$. The additive noise on the output is coloured and expressed as

$$
v(t)=H(p) e(t),
$$

where $H(p)$ is an inversely stable filter and $e(t)$ a zeromean Gaussian noise, i.e. $e \sim N\left(0, \sigma^{2}\right)$. The output ob- servation equation of the CT system (1) at sample instance $t_{k}$ is given by

$$
\stackrel{\circ}{y}\left(t_{k}\right)=\stackrel{\circ}{x}\left(t_{k}\right)+v\left(t_{k}\right),
$$

where $\stackrel{x}{x}\left(t_{k}\right)$ is the unobserved, noise-free output. It is well known that CT white noise does not have a finite variance [2], which makes computing its time-derivatives particularly difficult. Due to this difficulty and the DT nature of the sampled signals, we only consider DT noise in this paper. The true system and the nature of sampling are shown in Fig. 1.

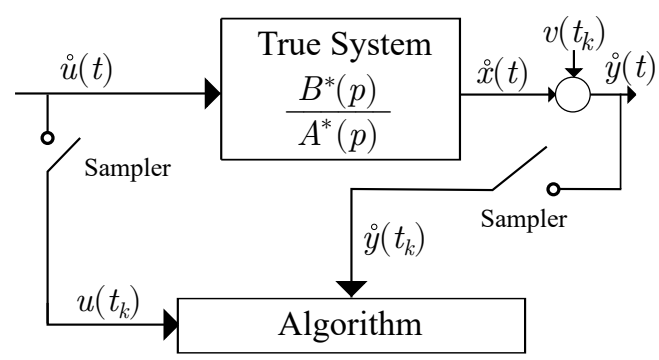

Fig. 1. Continuous-time system including the sampling and signal notations.

The model is also parameterised as a proper transfer function

$$
M:\left\{\begin{array}{l}
x\left(t_{k}\right)=\frac{B(p)}{A(p)} u\left(t_{k}\right) \\
y\left(t_{k}\right)=x\left(t_{k}\right)+e\left(t_{k}\right),
\end{array}\right.
$$

where $u\left(t_{k}\right)=\stackrel{\imath}{u}(t)$ at the sampling instants, and the numerator polynomial with degree $m$ and the denominator polynomial with degree $n$ are given by

$$
\begin{aligned}
& B(p)=b_{0} p^{m}+b_{1} p^{m-1}+\cdots+b_{m} \\
& A(p)=a_{1} p^{n}+a_{2} p^{n-1}+\cdots+a_{n} p+1 .
\end{aligned}
$$

The unknown parameter vector is then defined as

$$
\theta=\left[\begin{array}{llllll}
a_{1} & \ldots & a_{n} & b_{0} & \ldots & b_{m}
\end{array}\right]^{\top}
$$

Note that in the sequel when a mixed notation of CT operators and DT data is encountered in the analysis such as in (4), it implies that the input $u\left(t_{k}\right)$ in (4) is interpolated in some manner, e.g. using either a zeroorder or a first-order hold, and the resultant output is sampled at $t_{k}$.

The SRIVC estimator minimises the sum of squares of the generalised equation error (GEE) [22] $\varepsilon\left(t_{k}\right)$, which 
is given by

$$
\begin{aligned}
& \varepsilon\left(t_{k}\right)=\stackrel{\circ}{y}\left(t_{k}\right)-x\left(t_{k}\right) \\
& =\stackrel{\circ}{y}\left(t_{k}\right)-\frac{B(p)}{A(p)} u\left(t_{k}\right) \\
& =\frac{1}{A(p)}\left(A(p) \grave{y}\left(t_{k}\right)-B(p) u\left(t_{k}\right)\right) \\
& =A(p) y_{f}\left(t_{k}\right)-B(p) u_{f}\left(t_{k}\right) \text {, }
\end{aligned}
$$

where

$$
y_{f}\left(t_{k}\right)=\frac{1}{A(p)} \stackrel{y}{ }\left(t_{k}\right), \text { and } u_{f}\left(t_{k}\right)=\frac{1}{A(p)} u\left(t_{k}\right) .
$$

Due to the iterative nature of the SRIVC method, the $(j+1)$-th iteration of the SRIVC estimate $[29,8]$ based on parameters estimated in the $j$-th iteration is given by

$\theta_{j+1}=\left[\frac{1}{N} \sum_{k=1}^{N} \hat{\varphi}_{f}\left(t_{k}\right) \varphi_{f}^{\top}\left(t_{k}\right)\right]^{-1}\left[\frac{1}{N} \sum_{k=1}^{N} \hat{\varphi}_{f}\left(t_{k}\right) y_{f}\left(t_{k}\right)\right]$,

where

$$
\begin{aligned}
\varphi_{f}\left(t_{k}\right)=\frac{1}{A_{j}(p)}\left[-p^{n} \grave{y}\left(t_{k}\right)\right. & \ldots-p \grave{y}\left(t_{k}\right) \\
& \left.p^{m} u\left(t_{k}\right) \ldots u\left(t_{k}\right)\right]^{\top},
\end{aligned}
$$

and

$$
\begin{array}{r}
\hat{\varphi}_{f}\left(t_{k}\right)=\left[-x_{f}^{(n)}\left(t_{k}\right) \cdots-x_{f}^{(1)}\left(t_{k}\right) u_{f}^{(m)}\left(t_{k}\right) \ldots u_{f}\left(t_{k}\right)\right]^{\top} \\
=\frac{1}{A_{j}(p)}\left[-\frac{B_{j}(p)}{A_{j}(p)} p^{n} u\left(t_{k}\right) \ldots-\frac{B_{j}(p)}{A_{j}(p)} p u\left(t_{k}\right)\right. \\
\left.p^{m} u\left(t_{k}\right) \ldots u\left(t_{k}\right)\right]^{\top}
\end{array}
$$

The algorithm is stopped either when a maximum number of iterations is reached or when the relative error between the previous and current estimate is smaller than a prefixed constant, i.e.

$$
\frac{\left\|\theta_{j+1}-\theta_{j}\right\|}{\left\|\theta_{j+1}\right\|}<\epsilon .
$$

Next, we provide a definition of generic non-singularity [18] and relate it to the definition of generic consistency.

Definition 1 Consider an $n \times n$ matrix $R(\rho)$, which depends on a finite-dimensional vector $\rho$. Then, $R$ is generically non-singular with respect to $\rho$ if the set $\{\rho$ : $\operatorname{rank} R(\rho)<n\}$ has Lebesgue measure zero.

Definition 2 The SRIVC estimator (8) is generically consistent if the term in the matrix inverse in (8) is generically non-singular, and for all $j \geq 1$, the set of parameter values for which the estimates do not converge to the true parameters as the sample size tends to infinity has Lebesgue measure zero.

We note that all the filtering operations are performed in discrete-time within the implementation of the SRIVC estimator, hence the need to explicitly consider the intersample behaviour of the signals in any analysis. A block diagram depicting the SRIVC algorithm is shown in Fig. 2. In the following section, we investigate the effect of the intersample behaviour of the sampled data on the consistency of the SRIVC estimator. It will be shown that the input signal in the regressor vector, i.e. the model input, is required to have the same intersample behaviour as the input applied to the true system for the SRIVC estimator to be generically consistent. This intersample behaviour is circled in Fig. 2.

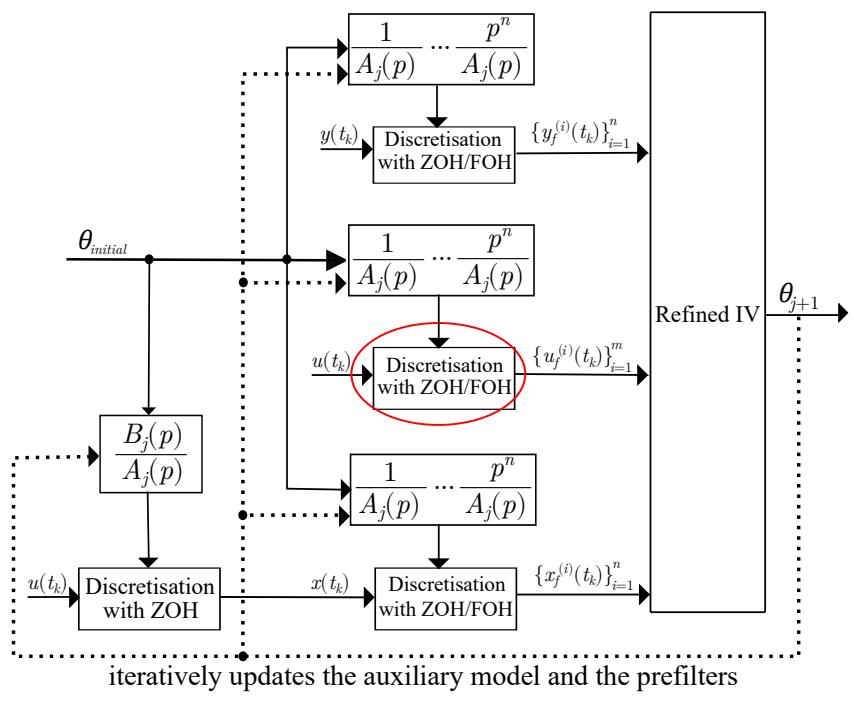

Fig. 2. Implementation of the SRIVC algorithm. Note that we use the notation $u^{(n)}\left(t_{k}\right)=p^{n} u\left(t_{k}\right)$.

\section{Theoretical Results}

In this section, we develop a theorem that establishes the consistency of the SRIVC estimator, as well as some corollaries and remarks that examine the consistency with respect to different intersample conditions. Some additional lemmas required by the proof of the consistency theorem are presented in the Appendix.

For simplicity, the analysis will be presented for the case of a single-input single-output (SISO), linear, time invariant (LTI), asymptotically stable system with regularly sampled data. We note that the analysis can be easily extended to multi-input single-output (MISO) systems, though the extension may be difficult for multiinput multi-output (MIMO) systems.

We next state the assumptions required in Theorem 1 for the SRIVC estimator to be generically consistent. 
Assumption 1 The true system $\frac{B^{*}(p)}{A^{*}(p)}$ is $\operatorname{proper}\left(n^{*} \geq\right.$ $\left.m^{*}\right)$ and asymptotically stable with $A^{*}(p)$ and $B^{*}(p)$ being coprime.

Assumption 2 The input $u\left(t_{k}\right)$ and disturbance $v\left(t_{s}\right)$ are stationary and mutually independent for all $k$ and $s$.

Assumption 3 The input $u\left(t_{k}\right)$ is persistently exciting of order no less than $2 n+1$.

Assumption 4 All the zeros of $A_{j}(p)$ have strictly negative real parts, $n \geq m$, with $A_{j}(p)$ and $B_{j}(p)$ being coprime.

Assumption 5 The degrees of the polynomials in the model satisfy $\min \left(n-n^{*}, m-m^{*}\right)=0$.

Assumption 6 The intersample behaviour of the input $\stackrel{u}{(t)}$ applied to the true system is known exactly.

Assumption 7 The sampling frequency is more than twice of the largest imaginary part of the zeros of $A_{j}(p) A^{*}(p)$.

When estimating the parameters of a transfer function, unstable zeros in the denominator polynomial may arise within the iterations of the algorithm. A simple way to deal with this is to reflect the unstable zeros by the imaginary axis. Hence, Assumption 4 is commonly satisfied in practice. Note that Assumption 5 ensures a unique solution for the model parameters [18]. Also note that in the proof of Theorem 1, we assume the intersample behaviour in Assumption 6 to be a FOH to conduct the analysis. We could have equally chosen to use a $\mathrm{ZOH}$ to model the intersample behaviour of the input as shown in Corollary 2. Furthermore, the prefilters for the output are assumed to be discretised with the same hold as the intersample behaviour of the input, i.e. a FOH in Theorem 1. Assumption 7 avoids the problem of aliasing and ensures a meaningful model to be obtained according to the Shannon-Nyquist theorem. Next, we present the main theorem of the paper on the generic consistency of the SRIVC estimator.

Theorem 1 (Generic consistency) Consider the SRIVC estimator described in (8), and suppose Assumptions 1-7 hold. Then, for a first-order hold (FOH) input, the following statements are true:

(1) The matrix $E\left\{\hat{\varphi}_{f}\left(t_{k}\right) \varphi_{f}^{\top}\left(t_{k}\right)\right\}$ is generically nonsingular.

(2) The true parameter $\theta^{*}$ is the unique converging point.

(3) As the sample size $N$ approaches infinity, $\theta_{j+1}$ in (8) converges to $\theta^{*}$ for $j \geq 1$.

Proof of Theorem 1, Statement 1. By substituting

$$
\stackrel{\circ}{y}\left(t_{k}\right)=\frac{B^{*}(p)}{A^{*}(p)} u\left(t_{k}\right)+v\left(t_{k}\right)
$$

into (9), we can express the regressor vector as

$$
\varphi_{f}\left(t_{k}\right)=\frac{1}{A_{j}(p) A^{*}(p)} Q_{r e g}-\frac{1}{A_{j}(p)} P_{r e g},
$$

where

$$
\begin{aligned}
Q_{r e g}=\left[-p^{n} B^{*}(p) u\left(t_{k}\right)\right. & \ldots-p B^{*}(p) u\left(t_{k}\right) \\
& \left.p^{m} A^{*}(p) u\left(t_{k}\right) \ldots A^{*}(p) u\left(t_{k}\right)\right]^{\top},
\end{aligned}
$$

and

$$
P_{r e g}=\left[\begin{array}{llllll}
p^{n} v\left(t_{k}\right) & \ldots & p v\left(t_{k}\right) & 0 & \ldots & 0
\end{array}\right]^{\top} .
$$

The highest order derivative in $Q_{r e g}$ that satisfies Assumption 5 is $\max \left(n+m^{*}, n^{*}+m\right)=n+m$. The vector $Q_{\text {reg }}$ can then be expressed as a product of an $(n+m+1) \times(n+m+1)$ Sylvester matrix and a vector containing the derivatives of $u\left(t_{k}\right)$, i.e.

$$
Q_{r e g}=S\left(-B^{*}(p), A^{*}(p)\right) U_{d u}
$$

where

$S\left(-B^{*}(p), A^{*}(p)\right)=\left[\begin{array}{ccccccc}-b_{0}^{*} & -b_{1}^{*} & \cdots & -b_{m^{*}}^{*} & 0 & & 0 \\ & \ddots & & & \ddots & \ddots & \\ & & -b_{0}^{*} & -b_{1}^{*} & \cdots & -b_{m^{*}}^{*} & 0 \\ \hline 0 & & & & & \\ \hline a_{1}^{*} & a_{2}^{*} & \cdots & a_{n^{*}}^{*} & 1 & & 0 \\ & \ddots & & & \ddots & \ddots & \\ 0 & & a_{1}^{*} & a_{2}^{*} & \cdots & a_{n^{*}}^{*} & 1\end{array}\right]$,

and

$$
U_{d u}=\left[u^{(n+m)}\left(t_{k}\right) u^{(n+m-1)}\left(t_{k}\right) \ldots u\left(t_{k}\right)\right]^{\top} .
$$

The Sylvester matrix given in (14) is non-singular when $B^{*}(p)$ and $A^{*}(p)$ are coprime [18]. We require (14) to remain non-singular under the three conditions imposed by Assumption 5, i.e. 1) the order of the true system is known exactly, 2) the numerator of the model is overfitted, and 3) the denominator of the model is overfitted.

We note that there are $n$ rows of the numerator coefficients and $m+1$ rows of the denominator coefficients. Now, condition 1 corresponds to (14). Under condition 2, when the numerator polynomial is overfitted, i.e. $m-m^{*}=l$ for all positive integers $l$, the first $l$ columns of the top half of (14) are filled with zeros. Similarly, under condition $3, n-n^{*}=l$ for all positive integers $l$, the first $l$ columns of the bottom half of (14) are filled with zeros. Nevertheless, in all three cases, (14) does not lose rank since it is guaranteed that there is at least one non-zero entry in each column due to the non-monic model denominator assumption. Therefore, $S\left(-B^{*}(p), A^{*}(p)\right)$ is non-singular under Assumption 5 . 
Now, the regressor vector in (12) can be written as

$$
\varphi_{f}\left(t_{k}\right)=\frac{S\left(-B^{*}(p), A^{*}(p)\right)}{A_{j}(p) A^{*}(p)} U_{d u}-\frac{1}{A_{j}(p)} P_{r e g} .
$$

Similarly, the instrument vector in (8) can be written as

$$
\hat{\varphi}_{f}\left(t_{k}\right)=\frac{S\left(-B_{j}(p), A_{j}(p)\right)}{A_{j}^{2}(p)} U_{d u},
$$

where $S\left(-B_{j}(p), A_{j}(p)\right)$ is an $(n+m+1) \times(n+$ $m+1)$ Sylvester matrix defined in the same way as (14) with $a_{1}^{*}, \ldots, a_{n^{*}}^{*}, b_{0}^{*}, \ldots, b_{m^{*}}^{*}$ replaced by $a_{1}, \ldots, a_{n}, b_{0}, \ldots, b_{m}$. From now on, we omit the argument $p$ in the Sylvester matrices for simplicity of notation.

It has been shown [15] that, as $N \rightarrow \infty$, the sums in (8) can be replaced by their expectations, provided $\hat{\varphi}_{f}\left(t_{k}\right)$, $\varphi_{f}^{\top}\left(t_{k}\right)$ and $y_{f}\left(t_{k}\right)$ are jointly stationary stochastic processes. Now, substituting (16) and (17) into the matrix inverse term in (8), we obtain

$$
\begin{aligned}
& E\left\{\hat{\varphi}_{f}\left(t_{k}\right) \varphi_{f}^{\top}\left(t_{k}\right)\right\} \\
& \quad=S\left(-B_{j}, A_{j}\right) \Phi S^{\top}\left(-B^{*}, A^{*}\right)-S\left(-B_{j}, A_{j}\right) \Psi
\end{aligned}
$$

where

$$
\Phi=E\left\{\frac{1}{A_{j}^{2}(p)} U_{d u} \frac{1}{A_{j}(p) A^{*}(p)} U_{d u}^{\top}\right\},
$$

and

$$
\Psi=E\left\{\frac{1}{A_{j}^{2}(p)} U_{d u} \frac{1}{A_{j}(p)} P_{r e g}^{\top}\right\} .
$$

According to Lemma A3.1 in [18], the Sylvester matrices $S\left(-B_{j}, A_{j}\right)$ and $S\left(-B^{*}, A^{*}\right)$ are non-singular provided that $B_{j}(p)$ and $A_{j}(p)$ are coprime and $B^{*}(p)$ and $A^{*}(p)$ are coprime. For (18) to be non-singular, it is sufficient to show that $\Phi$ is non-singular, and $\Psi=0$.

Consider $\Psi$ in (20). An arbitrary entry in the first $n$ columns of $\Psi$ can be written in the form of

$$
\begin{aligned}
\Psi_{i l} & =E\left\{\frac{p^{n+m+1-i}}{A_{j}^{2}(p)} u\left(t_{k}\right) \frac{p^{n+1-l}}{A_{j}(p)} v\left(t_{k}\right)\right\} \\
& =\frac{1}{2 \pi} \int_{-\pi}^{\pi} \frac{\tilde{B}_{i}\left(e^{j \omega}\right)}{\tilde{A}_{j}^{2}\left(e^{j \omega}\right)} \frac{\tilde{D}_{l}\left(e^{-j \omega}\right)}{\tilde{A}_{j}\left(e^{-j \omega}\right)} \phi_{u v}(\omega) d \omega,
\end{aligned}
$$

where $\tilde{B}_{i} / \tilde{A}_{j}^{2}$ and $\tilde{D}_{l} / \tilde{A}_{j}$ are the FOH equivalents of their CT transfer functions respectively, $i=1, \ldots, n+m+1$, $l=1, \ldots, n$, and $\phi_{u v}(\omega)$ is the cross-spectrum of $u\left(t_{k}\right)$ and $v\left(t_{k}\right)$. Since the input and noise are uncorrelated, $\phi_{u v}(\omega)=0$. Thus, $\Psi=0$.

Now, consider $\Phi$ in (19). Similarly, an arbitrary entry of this matrix can be written as

$$
\begin{aligned}
\Phi_{i l} & =E\left\{\frac{p^{n+m+1-i}}{A_{j}^{2}(p)} u\left(t_{k}\right) \frac{p^{n+m+1-l}}{A_{j}(p) A^{*}(p)} u\left(t_{k}\right)\right\} \\
& =\frac{1}{2 \pi} \int_{-\pi}^{\pi} \frac{\tilde{B}_{i}\left(e^{j \omega}\right)}{\tilde{A}_{j}^{2}\left(e^{j \omega}\right)} \frac{\tilde{B}_{l}\left(e^{-j \omega}\right)}{\tilde{A}_{j}\left(e^{-j \omega}\right) \tilde{A}^{*}\left(e^{-j \omega}\right)} d F_{u}(\omega),
\end{aligned}
$$

where $i, l=1, \ldots, n+m+1$, and $F_{u}(\omega)$ is the spectral distribution of $u\left(t_{k}\right)$.

We have shown in Lemma 7 (see Appendix) that $\Phi$ is positive definite when evaluated at the true system parameters. By Lemma 9 (see Appendix), we have also shown that for a fixed input signal, every entry of $\Phi$ is an analytic function of the model parameters. Hence, by Lemma A2.3 of [18], we can conclude that $\Phi$ is generically non-singular. Since $S\left(-B_{j}, A_{j}\right)$ and $S\left(-B^{*}, A^{*}\right)$ are non-singular, and $\Psi=0, E\left\{\hat{\varphi}_{f}\left(t_{k}\right) \varphi_{f}^{\top}\left(t_{k}\right)\right\}$ is generically non-singular.

Proof of Theorem 1, Statement 2. Here we will show that, upon convergence, the limiting point of the SRIVC estimator corresponds to the true parameters. Suppose $\bar{\theta}$ is a limiting point of the iteration in (8), and the corresponding polynomials of the model are given by

$$
\begin{aligned}
& \bar{B}(p)=\bar{b}_{0} p^{m}+\bar{b}_{1} p^{m-1}+\cdots+\bar{b}_{m} \\
& \bar{A}(p)=\bar{a}_{1} p^{n}+\bar{a}_{2} p^{n-1}+\cdots+\bar{a}_{n} p+1 .
\end{aligned}
$$

The polynomials $\bar{B}(p)$ and $\bar{A}(p)$ are coprime since $\bar{\theta}$ satisfies the conditions in Statement 1, and one of the conditions is that the Sylvester matrix is non-singular. Now, at the converging point $\bar{\theta}$, as $N \rightarrow \infty$, the SRIVC expression in (8) implies that

$$
E\left\{\hat{\varphi}_{f}\left(t_{k}, \bar{\theta}\right) \varphi_{f}^{\top}\left(t_{k}, \bar{\theta}\right)\right\}^{-1} E\left\{\hat{\varphi}_{f}\left(t_{k}, \bar{\theta}\right) \varepsilon\left(t_{k}, \bar{\theta}\right)\right\}=0,
$$

where $\varepsilon\left(t_{k}, \bar{\theta}\right)$ is the GEE evaluated at the converging point. Since the matrix inverse in (23) is non-singular by Statement 1, the second expectation in (23) must be zero, i.e.

$$
E\left\{\hat{\varphi}\left(t_{k}, \bar{\theta}\right) \varepsilon\left(t_{k}, \bar{\theta}\right)\right\}=0 .
$$

The GEE in (6) can be rearranged as

$$
\begin{array}{r}
\varepsilon\left(t_{k}, \bar{\theta}\right)=\frac{1}{\bar{A}(p) A^{*}(p)}\left[\bar{A}(p) B^{*}(p)-\bar{B}(p) A^{*}(p)\right] u\left(t_{k}\right) \\
+v\left(t_{k}\right) .
\end{array}
$$


Let $\bar{A}(p) B^{*}(p)-\bar{B}(p) A^{*}(p)=h_{0} p^{r}+h_{1} p^{r-1}+\cdots+h_{r}$, where $r=\max \left(n+m^{*}, n^{*}+m\right)=n+m$. Then, the GEE can be expressed as

$\varepsilon\left(t_{k}, \bar{\theta}\right)=\frac{1}{\bar{A}(p) A^{*}(p)}\left[u^{(n+m)}\left(t_{k}\right) \ldots u\left(t_{k}\right)\right] H+v\left(t_{k}\right)$,

where

$$
H=\left[\begin{array}{llll}
h_{0} & h_{1} & \ldots & h_{n+m}
\end{array}\right]^{\top} .
$$

Now, substituting (17) for $\hat{\varphi}\left(t_{k}, \bar{\theta}\right)$ and $(26)$ for $\varepsilon\left(t_{k}, \bar{\theta}\right)$ into (24), we obtain

$$
E\left\{\hat{\varphi}\left(t_{k}, \bar{\theta}\right) \varepsilon\left(t_{k}, \bar{\theta}\right)\right\}=S(-\bar{B}, \bar{A}) \bar{\Phi} H+S(-\bar{B}, \bar{A}) \tilde{\Psi},
$$

where $\bar{\Phi}$ is (19) evaluated at the converging point, and

$$
\tilde{\Psi}=E\left\{\frac{1}{\bar{A}^{2}(p)} U_{d u} v\left(t_{k}\right)\right\} .
$$

By following the same procedure as the proof of Statement 1 , we can show that $\bar{\Phi}$ is generically non-singular and $\tilde{\Psi}=0$. Thus, for (28) to be zero, $H=0$, which implies

$$
\begin{gathered}
\bar{A}(p) B^{*}(p)-\bar{B}(p) A^{*}(p)=0 \\
\frac{\bar{B}(p)}{\bar{A}(p)}=\frac{B^{*}(p)}{A^{*}(p)},
\end{gathered}
$$

i.e. $\theta^{*}$ is the unique limiting point.

Proof of Theorem 1, Statement 3. Let $\bar{\theta}$ be the limiting point, then, as $N \rightarrow \infty$,

$$
\theta_{j+1}-\bar{\theta}=f_{1}\left(\theta_{j}\right) f_{2}\left(\theta_{j}\right)
$$

where

$$
f_{1}\left(\theta_{j}\right)=E\left\{\hat{\varphi}_{f}\left(t_{k}, \theta_{j}\right) \varphi_{f}^{\top}\left(t_{k}, \theta_{j}\right)\right\}^{-1},
$$

and

$$
f_{2}\left(\theta_{j}\right)=E\left\{\hat{\varphi}_{f}\left(t_{k}, \theta_{j}\right)\left(y_{f}\left(t_{k}, \theta_{j}\right)-\varphi_{f}^{\top}\left(t_{k}, \theta_{j}\right) \bar{\theta}\right)\right\} .
$$

To examine how the SRIVC estimate behaves around the limiting point, we can linearise $\theta_{j+1}$ around $\bar{\theta}$ using a first order Taylor series, i.e.

$$
\begin{aligned}
\theta_{j+1}-\bar{\theta} \approx f_{1}(\bar{\theta}) f_{2}(\bar{\theta}) & +\left(\left.\frac{\partial f_{1}\left(\theta_{j}\right)}{\partial \theta_{j}}\right|_{\theta_{j}=\bar{\theta}} f_{2}(\bar{\theta})\right. \\
& \left.+\left.f_{1}(\bar{\theta}) \frac{\partial f_{2}\left(\theta_{j}\right)}{\partial \theta_{j}}\right|_{\theta_{j}=\bar{\theta}}\right)\left(\theta_{j}-\bar{\theta}\right) .
\end{aligned}
$$

At the limiting point, $f_{2}(\bar{\theta})=0$ as given by $(24)$. Hence,

$$
\theta_{j+1}-\left.\bar{\theta} \approx f_{1}(\bar{\theta}) \frac{\partial f_{2}\left(\theta_{j}\right)}{\partial \theta_{j}}\right|_{\theta_{j}=\bar{\theta}}\left(\theta_{j}-\bar{\theta}\right)
$$

where

$$
\begin{gathered}
\left.\frac{\partial f_{2}\left(\theta_{j}\right)}{\partial \theta_{j}}\right|_{\theta_{j}=\bar{\theta}}=E\left\{\left.\frac{\partial \hat{\varphi}_{f}\left(t_{k}, \theta_{j}\right)}{\partial \theta_{j}}\right|_{\theta_{j}=\bar{\theta}}\left(y_{f}\left(t_{k}, \bar{\theta}\right)-\varphi_{f}^{\top}\left(t_{k}, \bar{\theta}\right) \bar{\theta}\right)\right\} \\
+E\left\{\hat{\varphi}_{f}\left(t_{k}, \bar{\theta}\right)\left(\left.\frac{\partial y_{f}\left(t_{k}, \theta_{j}\right)}{\partial \theta_{j}}\right|_{\theta_{j}=\bar{\theta}}-\left.\frac{\partial \varphi_{f}^{\top}\left(t_{k}, \theta_{j}\right)}{\partial \theta_{j}}\right|_{\theta_{j}=\bar{\theta}} \bar{\theta}\right)\right\} \\
=\Psi_{1}+\Psi_{2} .
\end{gathered}
$$

After some vector differentiations and substituting the expression

$$
\begin{aligned}
y_{f}\left(t_{k}, \bar{\theta}\right)-\varphi_{f}^{\top}\left(t_{k}, \bar{\theta}\right) \bar{\theta} & =y_{f}\left(t_{k}, \theta^{*}\right)-\varphi_{f}^{\top}\left(t_{k}, \theta^{*}\right) \theta^{*} \\
& =v\left(t_{k}\right)
\end{aligned}
$$

into (30), we can express $\Psi_{1}$ as

$$
\Psi_{1}=E\left\{M u\left(t_{k}\right) v\left(t_{k}\right)\right\},
$$

where

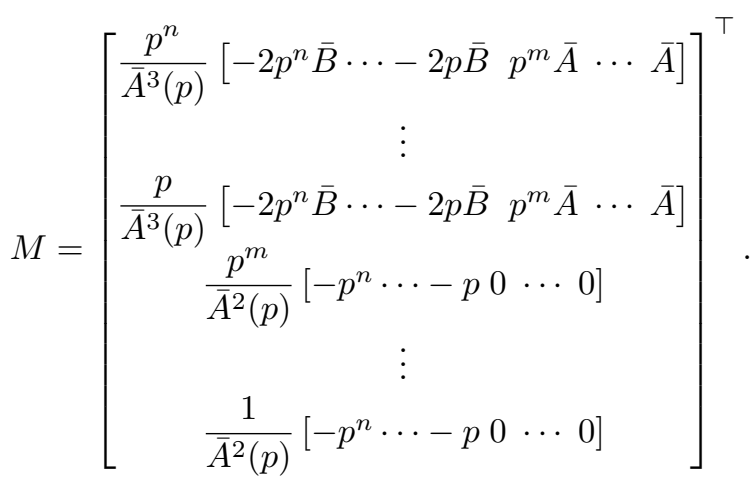

Similar to the procedure undertaken in Statement 1, each element of $\Psi_{1}$ can be expressed as

$$
\frac{1}{2 \pi} \int_{-\pi}^{\pi} \tilde{G}\left(e^{j \omega}\right) \phi_{u v}(\omega) d \omega
$$

where $\tilde{G}$ represents the FOH equivalent of the transfer functions in (31). Since the input and noise are uncorrelated, $\Phi_{u v}=0$. Hence, $\Psi_{1}=0$.

Now, after some further vector differentiations, $\Psi_{2}$ in (30) can be written as

$$
\begin{aligned}
\Psi_{2} & =-S(-\bar{B}, \bar{A}) E\left\{\frac{1}{\bar{A}^{2}(p)} U_{d u} \frac{1}{\bar{A}(p)} P_{r e g}^{\top}\right\} \\
& =-\bar{\Psi} \\
& =0
\end{aligned}
$$


where $\bar{\Psi}$ is (20) evaluated at the converging point. Hence, (30) is equal to zero. Therefore, according to $(29), \theta_{j+1}$ asymptotically converges to $\theta^{*}$ for $j \geq 1$, and this completes the final part of the proof.

Corollary 2 When the FOH used in Theorem 1 is replaced with a ZOH, and the intersample behaviour of the true system input $\stackrel{\sim}{u}(t)$ satisfies Assumption 6 , statements 1, 2 and 3 in Theorem 1 still hold.

Proof of Corollary 2. The proof follows the same procedure as that shown in Theorem 1. Note that, in this case, when the system and model transfer functions are strictly proper, the numerator degree of the DT transfer function is at most $n-1$. Thus, the persistent excitation order in Assumption 3 can be relaxed to $2 n$ according the reasoning provided in Remark 8 (see Appendix).

Theorem 1 and Corollary 2 have established consistency when the intersample behaviours of the input signals in both the regressor and instrument vectors as well as the output signal are assumed to be the same as that of the true system input. Next, Corollary 3 examines the effect on the consistency of the SRIVC estimates when an incorrect intersample behaviour is assumed for 1) the input in the instrument vector, and 2) the input in the regressor vector (the model input). Again, the true system input $\stackrel{u}{u}(t)$ is assumed to have an FOH for discretisation purposes, and an incorrect intersample behaviour means that the signal has an intersample behaviour that is different from the true system input.

\section{Corollary 3 The SRIVC estimator;}

(1) remains generically consistent if an incorrect assumption on the intersample behaviour is used for generating the filtered signals in the instrument vector $\hat{\varphi}_{f}\left(t_{k}\right)$ (this includes the generation of the noisefree model output $x\left(t_{k}\right)$, the filtered noise-free model output $x_{f}\left(t_{k}\right)$, and the filtered input signal $u_{f}\left(t_{k}\right)$ in (10)); and

(2) is generically not consistent if an incorrect assumption on the intersample behaviour is used for filtering the input signal in the regressor vector $\varphi_{f}\left(t_{k}\right)$.

Proof of Corollary 3, Statement 1. The input used to form the instrument vector is assumed to have a ZOH. Statement 1 of Theorem 1 still holds since the only change is that the $\mathrm{FOH}$ discretisations of the first transfer functions in (21) and (22) are replaced by their $\mathrm{ZOH}$ equivalents, and this does not affect the way analyticity of $\Psi$ is shown. Statement 2 in Theorem 1 remains unchanged since the incorrect intersample behaviour assumption of the input in the instrument does not affect the formulation of the equation error in (25). For the same reason, Statement 3 of Theorem 1 also remains unchanged. Therefore, when an incorrect intersample behaviour for the input signal in the instrument vector $\hat{\varphi}_{f}\left(t_{k}\right)$ is assumed, the SRIVC estimator remains generically consistent.

Proof of Corollary 3, Statement 2. Let the input in the regressor vector, indicated by $\tilde{u}\left(t_{k}\right)$, have a different intersample behaviour from that of the true system input. Statement 1 of Theorem 1 remains unchanged, however, we will show that Statement 2 of Theorem 1 has been affected. Consider the GEE at the limiting solution $\bar{\theta}$

$$
\begin{aligned}
& \varepsilon\left(t_{k}, \bar{\theta}\right)=\stackrel{\circ}{y}\left(t_{k}\right)-\frac{\bar{B}(p)}{\bar{A}(p)} \tilde{u}\left(t_{k}\right) \\
& =\frac{B^{*}(p)}{A^{*}(p)} u\left(t_{k}\right)+v\left(t_{k}\right)-\frac{\bar{B}(p)}{\bar{A}(p)} \tilde{u}\left(t_{k}\right) \\
& =\frac{B^{*}(p)}{A^{*}(p)} u\left(t_{k}\right)+v\left(t_{k}\right)-\left(\frac{\bar{B}(p)}{\bar{A}(p)} u\left(t_{k}\right)+\varepsilon_{u}\left(t_{k}\right)\right) \\
& =\frac{1}{\bar{A}(p) A^{*}(p)}\left[\bar{A}(p) B^{*}(p)-\bar{B}(p) A^{*}(p)\right] u\left(t_{k}\right) \\
& +v\left(t_{k}\right)-\varepsilon_{u}\left(t_{k}\right) .
\end{aligned}
$$

We have introduced an input-dependent term $\varepsilon_{u}\left(t_{k}\right)$ into the modelled output in (32) to account for the interpolation error. At the limiting point, (24) holds. Substituting (17) for $\hat{\varphi}_{f}\left(t_{k}, \bar{\theta}\right)$ and (32) for $\varepsilon\left(t_{k}, \bar{\theta}\right)$ into (24). By using the same definition of $H$ from (27), we obtain

$$
\begin{aligned}
& E\left\{\hat{\varphi}\left(t_{k}, \bar{\theta}\right) \varepsilon\left(t_{k}, \bar{\theta}\right)\right\} \\
& \quad=S(-\bar{B}, \bar{A}) \bar{\Phi} H-S(-\bar{B}, \bar{A}) E\left\{\frac{1}{\bar{A}^{2}(p)} U_{d u} \varepsilon_{u}\left(t_{k}\right)\right\} \\
& \quad=S(-\bar{B}, \bar{A}) \bar{\Phi} H-S(-\bar{B}, \bar{A}) \tilde{\Psi}_{u} \\
& \quad=0 .
\end{aligned}
$$

Since the error $\varepsilon_{u}\left(t_{k}\right)$ is input dependent, $\tilde{\Psi}_{u}$ does not go to zero in general. The matrix $\bar{\Phi}$ is generically nonsingular by Statement 1 of Theorem 1 . Therefore, we can obtain the coefficients of $H(p)$ by solving

$$
H=[S(-\bar{B}, \bar{A}) \bar{\Phi}]^{-1} S(-\bar{B}, \bar{A}) \tilde{\Psi}_{u}
$$

Now,

$$
\begin{aligned}
\bar{A}(p) B^{*}(p) & -\bar{B}(p) A^{*}(p)=H(p) \\
\frac{\bar{B}(p)}{\bar{A}(p)} & =\frac{B^{*}(p)}{A^{*}(p)}+\frac{H(p)}{\bar{A}(p) A^{*}(p)},
\end{aligned}
$$

where the parameters of the polynomial $H(p)$ are given by (34). The expression in (35) shows that the true parameters are no longer the limiting solution of the SRIVC estimator, i.e. $\bar{\theta} \neq \theta^{*}$. 
It is implied by (24) that the input is uncorrelated with the GEE evaluated at the converging point. Therefore, $\theta_{j+1}$ asymptotically converges to the new limiting point $\bar{\theta}$ given in (35) for $j \geq 1$. Together with $\bar{\theta} \neq \theta^{*}$, we can conclude that $\theta_{j+1}$ does not converge to the true parameters $\theta^{*}$ if an incorrect intersample behaviour is assumed for the input signal in the regressor vector $\varphi_{f}\left(t_{k}\right)$. Hence, the SRIVC estimator is generically not consistent when the true system input cannot be interpolated exactly.

For discretisation purposes, the true system input is assumed to have an FOH for the analysis above. We note that Corollary 3 holds for any input that cannot be interpolated exactly, as stated in the following remark.

Remark 4 When the input to the real system $\stackrel{u}{u}(t)$ is a continuous function of time that cannot be interpolated exactly, the SRIVC estimator is not consistent. This follows from Corollary 3.

We note that in situations where the input cannot be interpolated exactly, $\varepsilon_{u}\left(t_{k}\right)$ will be non-zero, and the bias on the estimates is captured by $\frac{H(p)}{A(p) A^{*}(p)}$ in (35). Since the polynomial $H(p)$ is proportional to the interpolation error $\varepsilon_{u}\left(t_{k}\right)$, which will decrease if the signals are sampled faster, this implies that the bias on the estimates will generally decrease with the sampling period.

Next, we examine the effect of the intersample behaviour of the sampled output on the consistency of the SRIVC estimator in the following remark.

Remark 5 Consider the GEE $\varepsilon\left(t_{k}\right)$. At each SRIVC iteration $j, \varepsilon\left(t_{k}\right)$ can be expressed as

$$
\begin{aligned}
& \varepsilon\left(t_{k}, \theta_{j}\right) \\
& \quad=A_{j}(p)\left(\frac{1}{A_{j-1}(p)} \stackrel{\circ}{y}\left(t_{k}\right)\right)-B_{j}(p)\left(\frac{1}{A_{j-1}(p)} u\left(t_{k}\right)\right) .
\end{aligned}
$$

Upon convergence, the expression in (36) becomes

$$
\varepsilon\left(t_{k}, \bar{\theta}\right)=\bar{A}(p)\left(\frac{1}{\bar{A}(p)} \stackrel{\circ}{y}\left(t_{k}\right)\right)-\bar{B}(p)\left(\frac{1}{\bar{A}(p)} u\left(t_{k}\right)\right) .
$$

Hence, the intersample behaviour of the measured output $\stackrel{y}{y}\left(t_{k}\right)$ does not affect the GEE as the discretisation of $1 / \bar{A}(p)$ cancels with that of $\bar{A}(p)$ at the converging point, and thus it does not influence the consistency of the SRIVC estimator.

Note it has been empirically observed that even though the intersample behaviour of $\stackrel{y}{y}\left(t_{k}\right)$ does not affect the consistency of the SRIVC estimator, a better interpolation of this signal, e.g. using a $\mathrm{FOH}$ as opposed to a $\mathrm{ZOH}$, can speed up the rate of convergence of the SRIVC iteration.

\section{Simulation Results}

Monte Carlo simulations are performed for a second order system to support the theoretical analyses developed in the previous section. The second order system is chosen to be

$$
G^{*}(p)=\frac{1}{0.04 p^{2}+0.2 p+1},
$$

and the true parameters are given by

$$
\theta^{*}=\left[\begin{array}{lll}
0.04 & 0.2 & 1
\end{array}\right]^{\top}
$$

The measured signals are sampled at $T=0.1 \mathrm{~s}$, and the input is chosen to be a random binary signal uniformly exciting the system from $0 \mathrm{~Hz}$ up to the Nyquist frequency. The input applied to the true system $\stackrel{u}{u}(t)$ has a zero-order hold intersample behaviour. The additive noise on the output is an i.i.d. Gaussian sequence with a variance of 0.1 . The consistency of the SRIVC estimator is investigated by examining the mean and variance of the estimates in a Monte Carlo simulation study as the sample size $N$ increases. Here, $N$ is adjusted from 50 to 200000 in a logarithmic scale, where a total of 100 different sample sizes are used. Three hundred Monte Carlo simulations are performed for each value of $N$ with the mean and variance of the three parameter estimates calculated. The maximum number of iterations of the SRIVC algorithm is set to 200 , and the relative error bound $\epsilon$ in (11) is set to $10^{-7}$. The mean and variance of the estimated parameters with respect to an increasing sample size are examined under four different cases by changing the intersample behaviour of the measured signals when discretising different filters in the SRIVC algorithm. These cases include

- matching the intersample behaviour of all the signals in the algorithm with that of $\stackrel{\circ}{u}(t)$, which is a $\mathrm{ZOH}$,

- setting only the intersample behaviour of $u\left(t_{k}\right)$ in the regressor vector to $\mathrm{FOH}$,

- setting only the intersample behaviour of $u\left(t_{k}\right)$ in the instrument vector to $\mathrm{FOH}$, and

- setting only the intersample behaviour of $\stackrel{y}{y}\left(t_{k}\right)$ to FOH.

These cases correspond to the first four instances in Fig. 3 and Fig. 4.

In another simulation, a multisine input, given by

$$
\stackrel{\circ}{u}(t)=\sin (0.5 t)+\sin (2 t)+\sin (5 t)+\sin (7 t),
$$

is used to excite the true system $G^{*}(p)$. The noiseless output is computed analytically by assuming that it corresponds to the system output at stationary state, i.e.

$$
x(t)=\sum_{i=1}^{4}\left|G^{*}\left(j \omega_{i}\right)\right| \sin \left(\omega_{i} t+\angle G^{*}\left(j \omega_{i}\right)\right)
$$


where $\left\{\omega_{1}, \omega_{2}, \omega_{3}, \omega_{4}\right\}=\{0.5,2,5,7\}$. The CT input and output are also sampled at $T=0.1 \mathrm{~s}$, and the additive noise on the measured output is an i.i.d. Gaussian sequence with a variance of 0.1. The same Monte Carlo studies as described previously for the random binary input are performed for the multisine input. The model input is interpolated using a FOH to approximate $\stackrel{u}{u}(t)$ as close as possible. The mean and variance of the estimates for each sample size are calculated to examine the consistency of the SRIVC estimator in situations when the input cannot be interpolated exactly. This corresponds to the fifth instance in Fig. 3 and Fig. 4.
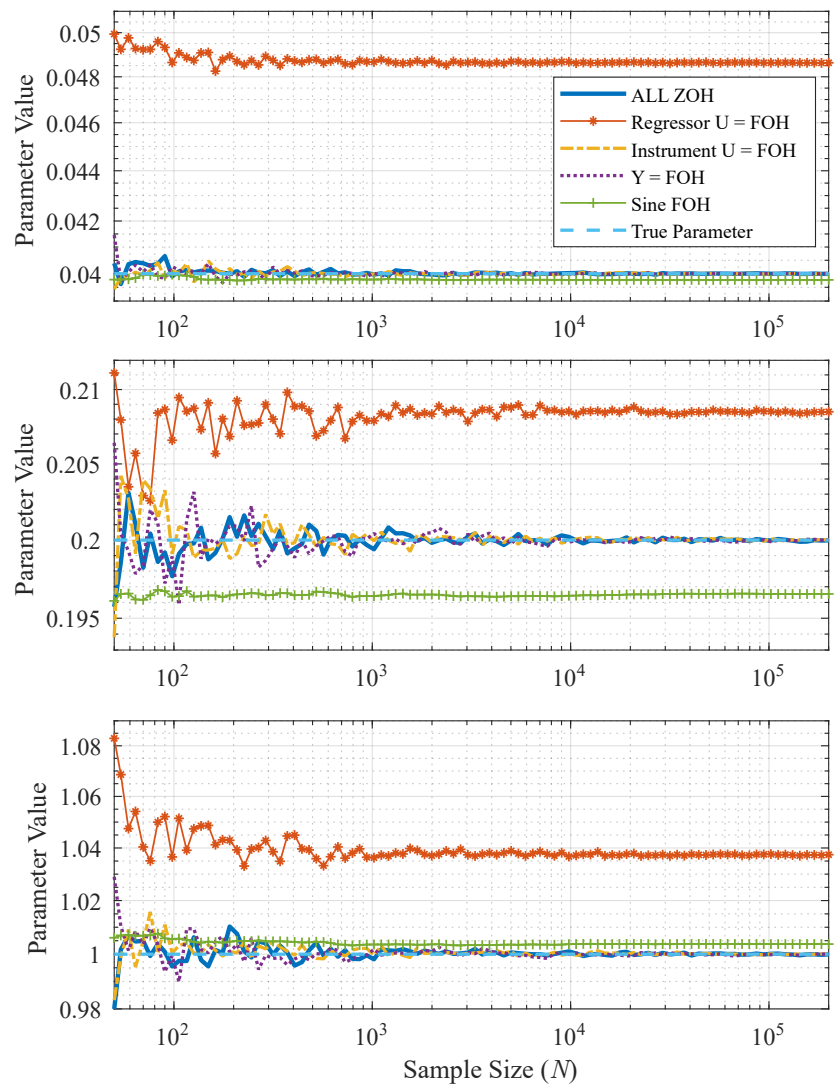

Fig. 3. Mean of the estimated parameters.

The mean and variance of the estimated parameters for the five instances described previously are shown in Fig. 3 and Fig. 4 respectively. The three subplots in both figures correspond to the parameters in the order of $0.04,0.2,1$. The true parameters are plotted with a dotted line in the three subplots of Fig. 3. We can see in Fig. 4 that the variance of the SRIVC estimates decreases with an increasing sample size in all cases. More oscillations in the mean values are observed for small sample sizes, but the estimates eventually converge to the true parameters after approximately 10000 samples in situations where we have matched the intersample behaviour of $u\left(t_{k}\right)$ in the regressor vector with that of the input applied to the true system, i.e. instances 1, 3 and 4. Together with the decreasing variance, this provides
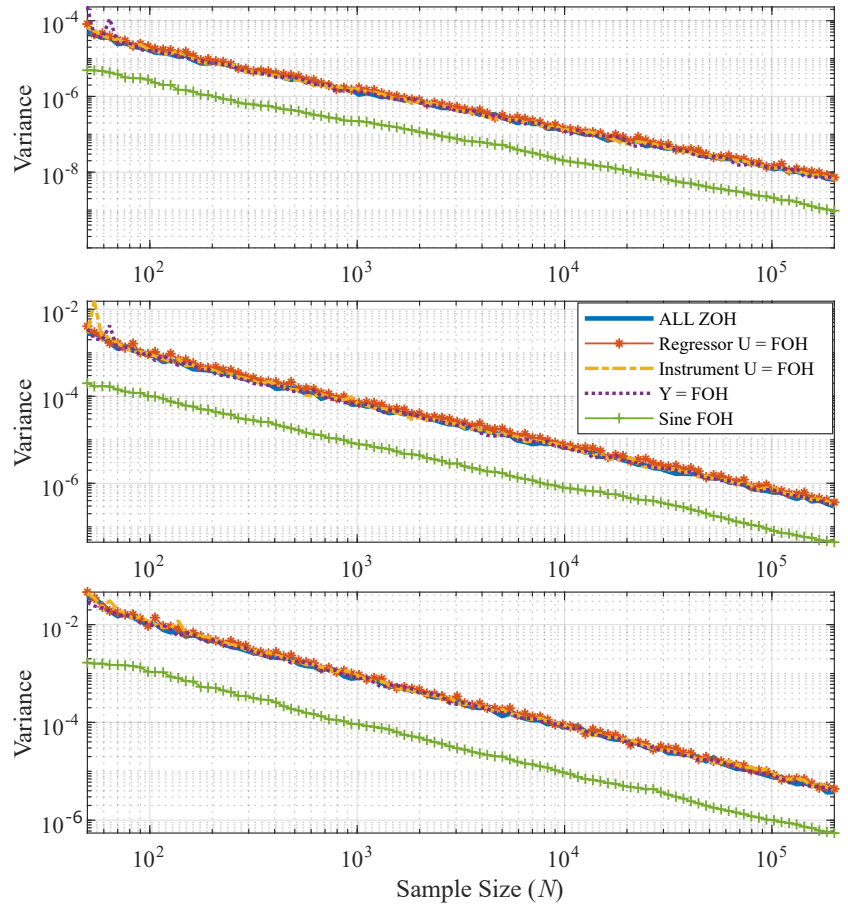

Fig. 4. Variance of the estimated parameters.

empirical evidence to the consistency result in Theorem 1 . In addition, changing the intersample behaviour of the input in the instrument vector or the output does not seem to affect the consistency of the SRIVC estimates, which aligns with statement 1 of Corollary 3 and Remark 5 respectively. We can also see that when the model input does not match the true system input, the estimates do not converge to the true parameters with an increasing sample size. The SRIVC estimator is not consistent in this case, which has been shown theoretically in statement 2 of Corollary 3. Furthermore, when a CT signal, which cannot be interpolated exactly, is used as the true system input, the SRIVC estimator can also be seen to be inconsistent. We do note that the bias on the SRIVC estimates can be reduced if more sophisticated interpolation methods other than a $\mathrm{ZOH}$ or $\mathrm{FOH}$ are used to reconstruct the input signal, and the bias will decrease with a decreasing sampling period as shown in statement 2 of Corollary 3.

\section{Conclusion}

In this paper, we have analysed the consistency of the SRIVC estimator by taking into account the intersample behaviour of the input signal, and conducted simulation experiments to provide empirical observations to the theoretical results. The main result of the paper is that the SRIVC estimator is generically consistent when the intersample behaviour of the input signal applied to the continuous-time system is known exactly and used adequately in the implementation of the algorithm. It 
has been shown that when the intersample behaviour of the input signal in the regressor, i.e. the model input, does not match that of the true system input, the unique converging point of the estimator no longer corresponds to the true parameters, and thus the SRIVC estimator is generically not consistent. On the other hand, the intersample behaviours of the input signal in the instrument vector or the output signal in the regressor vector do not affect the consistency of the estimator.

\section{Appendix}

Lemma 6 The mapping between the CT parameters $\left(a_{1}, \ldots, a_{n}\right)$ and the DT parameters $\left(\alpha_{1}, \ldots, \alpha_{n}\right)$ is analytic in $\left\{\left(a_{1}, \ldots, a_{n}\right) \in \mathbb{C}^{n}: a_{1} \neq 0\right\}$.

Proof of Lemma 6. Since $a_{1} \neq 0$, we can write

$$
\begin{aligned}
A^{\prime}(p) & :=p^{n}+\frac{a_{2}}{a_{1}} p^{n-1}+\cdots+\frac{a_{n}}{a_{1}} p+\frac{1}{a_{1}} \\
& =p^{n}+a_{1}^{\prime} p^{n-1}+\cdots+a_{n-1}^{\prime} p+a_{n}^{\prime}
\end{aligned}
$$

where we note that the mapping $\left(a_{1}, a_{2}, \ldots, a_{n}\right) \rightarrow$ $\left(a_{1}^{\prime}, a_{2}^{\prime}, \ldots, a_{n}^{\prime}\right)$ is analytic for $a_{1} \neq 0$. Let the state matrix of the CT system $1 / A^{\prime}(p)$ be $\mathrm{A}_{c}$. The state matrix of the DT equivalent is then $\mathrm{A}_{d}=\exp \left(\mathrm{A}_{c} T\right)$, where $T$ is the sampling period. The exponential is also analytic in the variables $\left(a_{1}^{\prime}, a_{2}^{\prime}, \ldots, a_{n}^{\prime}\right)$. Finally, we can express $\tilde{A}(z)=\operatorname{det}\left(z I-\mathrm{A}_{d}\right)$. It is known that the coefficients of this characteristic polynomial are polynomial expressions in the entries of the matrix $\mathrm{A}_{d}$ (see e.g. [4]). This implies that $\left\{\alpha_{i}\right\}_{i=1}^{n}$ are analytic functions of the entries of $\mathrm{A}_{d}$. The lemma then follows from the composition of analytic functions.

Lemma 7 Consider a FOH sampling, and Assumptions 1-7 hold. Then, the matrix $\Phi$ in (19) evaluated at the true system parameters, i.e.

$$
\Phi^{*}:=E\left\{\frac{1}{A^{*}(p)} U_{d u} \frac{1}{A^{* 2}(p)} U_{d u}^{\top}\right\}>0,
$$

is positive definite.

Proof of Lemma 7. Let $\mathbf{z} \in \mathbb{R}^{n+m+1}$. We can write

$$
\mathbf{z}^{\top} \Phi^{*} \mathbf{z}=E\left\{\left(\frac{B_{\mathbf{z}}(p)}{A^{*}(p)} u\left(t_{k}\right)\right)^{2}\right\} \geq 0
$$

where $B_{\mathbf{z}}(p)$ is an arbitrary polynomial of degree $n+m$. In the frequency domain, (38) can be written as

$$
\mathbf{z}^{\top} \Phi^{*} \mathbf{z}=\frac{1}{2 \pi} \int_{-\pi}^{\pi}\left|\frac{\tilde{B}_{\mathbf{z}}\left(e^{j \omega}\right)}{\tilde{A}^{*^{2}}\left(e^{j \omega}\right)}\right|^{2} d F_{u}(\omega)
$$

where $\tilde{B}_{\mathbf{z}}$ and $\tilde{A}^{*}$ are the FOH equivalent polynomials of $B_{\mathbf{z}}(p) / A^{* 2}(p)$, and $F_{u}(\omega)$ is the spectral distribution of $\left\{u\left(t_{k}\right)\right\}$. Note that $\tilde{B}_{\mathbf{z}}$ is in general a $2 n$ degree polynomial.

We can also write (38) as

$$
\mathbf{z}^{\top} \Phi^{*} \mathbf{z}=\frac{1}{2 \pi} \int_{-\pi}^{\pi}\left|\tilde{B}_{\mathbf{z}}\left(e^{j \omega}\right)\right|^{2} d F_{\tilde{u}}(\omega)
$$

where the support of the spectral distribution function $\phi_{\tilde{u}}$ consists of at least $2 n+1$ points since filtering $u\left(t_{k}\right)$ by $1 / \tilde{A}^{*^{2}}$ gives a signal which is also persistently exciting of order at least $2 n+1$. By the definition of persistence of excitation, $\mathbf{z}^{\top} \Phi^{*} \mathbf{z}=0$ implies $\tilde{B}_{\mathbf{z}}\left(e^{j \omega}\right) \equiv 0[12$, Theorem 1] and hence $\mathbf{z}$ is a zero vector. This means that $B_{\mathbf{z}}(p) / A^{*^{2}}(p)$ gives a sampled model equal to zero at all sampling instants.

Now, assume that there exists a $\mathbf{z}_{*}$ such that $\frac{B_{\mathbf{z}_{*}}(p)}{A^{* 2}(p)} u\left(t_{k}\right)=$ 0 . Thus, by linearity, for all $\mathbf{z}$ we have

$$
\frac{B_{\mathbf{z}}(p)}{A^{*^{2}}(p)} u\left(t_{k}\right)=\frac{B_{\mathbf{z}}(p)+B_{\mathbf{z}_{*}}(p)}{A^{*^{2}}(p)} u\left(t_{k}\right) .
$$

This means that, if $B_{\mathbf{z}_{*}}(p)$ were non-zero, the CT model is not uniquely determined by the DT model. However, this is not true under the sampling condition of the statement (see [10]). Therefore, it is not possible for a polynomial $B_{\mathbf{z}}(p)$ different from zero to give a sampled model equal to zero. This means that (38) is strictly positive for any non-zero vector $\mathbf{z}$. Hence, $\Phi^{*}$ is positive definite.

Remark 8 Note that even though there are $n+m+1$ parameters in the CT transfer function to be identified, the input is required to have a persistent excitation of order $2 n+1$ instead of $n+m+1$ for a first order hold discretisation of the input. The reason behind this is that the numerator polynomial $B_{z}(p)$ gets mapped to a subset of a larger space, namely a subset of the space of $2 n$ polynomials. Hence, singularity of $\Phi^{*}$ can be obtained by an unfortunate choice of the frequency lines of $\left\{u\left(t_{k}\right)\right\}$. If the input is persistently exciting of an order less than $2 n+1$, the frequency lines of the input could match the zeros of $\tilde{B}_{z}\left(e^{j \omega}\right)$, leading to $\boldsymbol{z}^{\top} \Phi^{*} \boldsymbol{z}=0$ when $\boldsymbol{z} \neq 0$.

Lemma 9 Each element of the matrix $\Phi$ in (19) is an analytic function of $a_{1}, \ldots, a_{n}$ for $\left(a_{1}, \ldots, a_{n}\right) \in \Omega$, where $\Omega$ denotes the subset of $\mathbb{C}^{n}$ consisting of parameter vectors $\left(a_{1}, \ldots, a_{n}\right)$ such that $A_{j}(p)$ has all zeros strictly in the left half-plane.

Proof of Lemma 9. Define the FOH equivalent of the model denominator as

$$
\tilde{A}_{j}(q):=q^{-n}+\alpha_{1} q^{-n+1}+\ldots+\alpha_{n}
$$


and denote $\Omega_{d}$ as the subset of $\mathbb{C}^{n}$ consisting of parameter vectors $\left(\alpha_{1}, \ldots, \alpha_{n}\right)$ such that $\tilde{A}_{j}(q)$ has all zeros strictly inside the unit circle. By Lemma 6 , there is an analytic mapping between $\left(a_{1}, \ldots, a_{n}\right)$ and the DT parameter vector $\left(\alpha_{1}, \ldots, \alpha_{n}\right)$. Now, fixing $\alpha_{2}, \ldots, \alpha_{n}$ allows us to define a region $\Omega_{d 1} \subset \mathbb{C}$ where $\bar{\alpha}_{1} \in \Omega_{d 1}$ implies $\left(\bar{\alpha}_{1}, \alpha_{2}, \ldots, \alpha_{n}\right) \in \Omega_{d}$. Note that the integrand in (22) is an analytic function of $\alpha_{1}$ in $\Omega_{d 1}$, and from now on, we denote this integrand as $f\left(\bar{\alpha}_{1}, \omega\right)$.

Let $C$ be a closed contour in $\Omega_{d 1}$ such that $\bar{\alpha}_{1}$ is interior to $C$. Then,

$$
f\left(\bar{\alpha}_{1}, \omega\right)=\frac{1}{2 \pi i} \int_{C} \frac{f\left(\alpha_{1}, \omega\right)}{\alpha_{1}-\bar{\alpha}_{1}} d \alpha_{1} .
$$

As a result,

$$
\Phi_{i l}\left(\bar{\alpha}_{1}\right)=\frac{1}{2 \pi} \int_{-\pi}^{\pi} \frac{1}{2 \pi i} \int_{C} \frac{f\left(\alpha_{1}, \omega\right)}{\alpha_{1}-\bar{\alpha}_{1}} d \alpha_{1} d F_{u}(\omega) .
$$

Since the function being integrated is bounded on $[-\pi, \pi] \times C$, the order of integration can be changed by Fubini's Theorem [19, p. 961], which yields

$$
\begin{aligned}
\Phi_{i l}\left(\bar{\alpha}_{1}\right) & =\frac{1}{2 \pi i} \int_{C} \frac{1}{\alpha_{1}-\bar{\alpha}_{1}} \frac{1}{2 \pi} \int_{-\pi}^{\pi} f\left(\alpha_{1}, \omega\right) d F_{u}(\omega) d \alpha_{1} \\
& =\frac{1}{2 \pi i} \int_{C} \frac{\Phi_{i l}\left(\alpha_{1}\right)}{\alpha_{1}-\bar{\alpha}_{1}} d \alpha_{1}
\end{aligned}
$$

from which we conclude that $\Phi_{i l}\left(\alpha_{1}\right)$ is analytic in a neighbourhood around $\alpha_{1}=\bar{\alpha}_{1}$.

Repeating this process for every $\alpha_{i}, i=2, \ldots, n$, we obtain that $\Phi_{i l}$ is an analytic function of the variables $\alpha_{1}, \alpha_{2}, \ldots, \alpha_{n}$ separately. Since $\Phi_{i l}$ is a continuous function of $\left(\alpha_{1}, \ldots, \alpha_{n}\right)$ in $\Omega_{d}, \Phi_{i l}$ is an analytic function of the joint variables $\left(\alpha_{1}, \ldots, \alpha_{n}\right)$ by Osgood's Lemma [3, p. 139]. Hence, each element of $\Phi$ is an analytic function of $a_{1}, \ldots, a_{n}$ for $\left(a_{1}, \ldots, a_{n}\right) \in \Omega$.

\section{References}

[1] T. Andersson, P. Pucar, and L. Ljung. Identification aspects of intersample input behavior. In IFAC System Identification, pages 1099 - 1104, 1994.

[2] K. J. Åström. Introduction to Stochastic Control Theory, chapter 2, pages $30-32$. Academic Press, 1970.

[3] S. Bochner and W. T. Martin. Several complex variables. Princeton Univ. Press, 1948.

[4] B. P. Brooks. The coefficients of the characteristic polynomial in terms of the eigenvalues and the elements of an $n \times n$ matrix. Applied mathematics letters, 19(6):511-515, 2006.

[5] F. Chen, M. Gilson, H. Garnier, and T. Liu. Robust timedomain output error method for identifying continuous-time systems with time delay. Systems and Control Letters, 102:81-92, 2017.
[6] C. T. Chou, M. Berhaegen, and R. Johansson. Continuoustime identification of siso systems using laguerre functions. IEEE Transactions on Signal Processing, 47(2):349 - 362, 1999.

[7] B. Finigan and I. Rowe. Strongly consistent parameter estimation by the introduction of strong instrumental variables. IEEE Transactions on Automatic Control, 19(6):825-830, 1974.

[8] H. Garnier and L. Wang, editors. Identification of Continuous-time Models from Sampled data. Springer, 2008.

[9] H. Garnier and P. C. Young. The advantages of directly identifying continuous-time transfer function models in practical applications. International Journal of Control, 2014.

[10] I. Kollar, G. Franklin, and R. Pintelon. On the equivalence of z-domain and s-domain models in system identification. In Instrumentation and Measurement Technology Conference, 1996, volume 1, pages 14-19, 1996.

[11] X. Liu, J. Wang, and W. X. Zheng. Convergence analysis of refined instrumental variable method for continuous-time system identification. IET Control Theory and Applications, 5(7):868-877, 2011.

[12] L. Ljung. Characterization of the concept of 'persistently exciting' in the frequency domain. Report TFRT, 3038, 1971.

[13] D. Marelli and M. Fu. A continuous-time linear system identification method for slowly sampled data. IEEE Transactions on Signal Processing, 58(5):2521 - 2533, 2010.

[14] J. Schoukens, R. Pintelon, and H. Van Hamme. Identification of linear dynamic systems using piecewise constant excitations: Use, misuse and alternatives. Automatica, 30(7):1153 - 1169, 1994

[15] T. Söderström. Ergodicity results for sample covariances. Problems of Control and Information Theory, 4(2):131-138, 1975.

[16] T. Söderström. Sampling approximations for continuous-time identification. In Proceedings of the 15th IFAC Symposium on System Identification, pages 1145 - 1150, 2009.

[17] T. Söderström and P. Stoica. Comparison of some instrumental variable methods - consistency and accuracy aspects. Automatica, 17(1):101-115, 1981.

[18] T. Söderström and P. Stoica. Instrumental Variable Methods for System Identification. Springer-Verlag, 1983.

[19] J. Stewart. Calculus: Early Transcendentals. Brooks/Cole, 6th edition, 2008.

[20] P. Stoica and T. Söderström. The Steiglitz-Mcbride identification algorithm revisited - convergence analysis and accuracy aspects. IEEE Transactions on Automatic Control, 26(3):712-717, 1981.

[21] P. Stoica and T. Söderström. Optimal instrumental variable estimation and approximate implementations. IEEE Transactions on Automatic Control, 28(7):757-772, 1983.

[22] P. C. Young. Parameter estimation for continuous-time models - a survey. Automatica, 17(1):23-39, 1981.

[23] P. C. Young. Optimal IV identification and estimation of continuous-time TF models. In 15th Triennial World Congress, volume 35, pages 109-114, 2002.

[24] P. C. Young. The refined instrumental variable method: Unified estimation of discrete and continuous-time transfer function models. Journal Europeen des Systemes Automatises, pages 149-179, 2008. 
[25] P. C. Young. Recursive estimation and time-series analysis: An introduction for the student and practitioner. SpringerVerlag, 2011.

[26] P. C. Young. Refined instrumental variable estimation: Maximum likelihood optimization of a unified box-jenkins model. Automatica, 52:35-46, 2015.

[27] P. C. Young and H. Garnier. Identification and estimation of continuous-time, data-based mechanistic models for environmental systems. Environmental Modelling \&
Software, 21(8):1055-1072, 2006.

[28] P. C. Young, H. Garnier, and M. Gilson. An optimal instrumental variable approach for identifying hybrid continuous-time box-jenkins models. In Proc. of the 14th IFAC Symposium on System Identification, volume 39, pages 225-230, 2006.

[29] P. C. Young and A. Jakeman. Refined instrumental variable methods of recursive time-series analysis part III. Extensions. International Journal of Control, 31(4):741-764, 1980. 